\title{
Cecal Insertion Time and the ADR: Patience Is Good for Patients
}

\author{
Yu-Hsi Hsieh ${ }^{1,2}$. Malcolm Koo Ko $^{3,4}$
}

Published online: 12 June 2018

(c) Springer Science+Business Media, LLC, part of Springer Nature 2018

The decline in incidence in colorectal cancer (CRC) over the past decades can be attributed in part to the success of colonoscopic screening and surveillance, since colonoscopy facilitates the identification and removal of precancerous lesions. The adenoma detection rate (ADR), which is defined as the proportion of patients with at least one identified adenoma, has emerged as one of the most important quality measures for colonoscopy. Colorectal cancers occurring after colonoscopy, i.e., interval cancers (ICs), are linked to a low ADR. A recent study showed that each $1 \%$ increase in ADR was associated with a $3 \%$ decrease in the risk of ICs [1].

An important quality improvement measure for maximizing ADR is to allow adequate time for inspection during colonoscope withdrawal. In a community-based study of 7882 colonoscopies, a mean withdrawal time $>6$ min was associated with a higher ADR [2]. Nevertheless, another study of 31,088 colonoscopies by 147 screening program colonoscopists indicated that the incremental increase in ADR was minimal when the withdrawal time was $>10 \mathrm{~min}$ [3]. Since not all colons are created equal, the withdrawal time needed for adequate inspection might vary among patients. Some patients have relatively straight colons that could be thoroughly inspected within $6 \mathrm{~min}$, whereas others have redundant and tortuous colons that require a longer withdrawal time.

Traditionally, cecal intubation time is considered a competency metric for colonoscopy. Many factors can influence

Yu-Hsi Hsieh

hsieh.yuhsi@msa.hinet.net

1 Division of Gastroenterology, Department of Medicine, Dalin Tzu Chi Hospital, Buddhist Tzu Chi Medical Foundation, 2 Minsheng Road, Dalin, Chiayi 62247, Taiwan

2 School of Medicine, Buddhist Tzu Chi University, Hualien, Taiwan

3 Department of Medical Research, Buddhist Dalin Tzu Chi Hospital, Chiayi, Taiwan

4 Dalla Lana School of Public Health, University of Toronto, Toronto, ON, Canada the cecal insertion time, which can be divided into two broad categories, namely patient-dependent and endoscopistdependent factors. Patient-dependent factors include sex, age, body mass index (or waist circumference), a history of constipation, and a history of prior abdominal surgery [4]. A longer insertion time typically reflects a more difficult examination, brought about by tortuous and acutely bending colons, which may subsequently hinder adenoma detection during withdrawal. For the endoscopist to speedily advance the endoscope to the cecum, proficient skills and efficient loop reduction are required, both of which may also help a meticulous search for adenoma during withdrawal. Conversely, after a prolonged insertion time, the endoscopist may feel fatigued and stressed for time, resulting in a hasty withdrawal with a higher chance of missing polyps.

Previous research reported an inverse association between cecal insertion time and ADR. Yang et al., in a crosssectional study of 12,679 consecutive study participants undergoing screening colonoscopy, reported that longer cecal insertion times were associated with lower detection rates of adenomas $<5 \mathrm{~mm}$, but not for adenomas $\geq 5 \mathrm{~mm}$, multiple adenomas, or advanced colorectal neoplasms [5]. Von Renteln et al. analyzed 1043 patients undergoing elective outpatient colonoscopy as part of a randomized controlled trial (RCT) on cap-assisted colonoscopy. They found that a longer cecal insertion time was linked to not only a decreased number of detected adenomas, but also advanced adenomas in the proximal and distal colons [6]. Moreover, in a study involving 10 endoscopists and 550 patients working in an academic center designed to assess the insertion timeto-withdrawal time ratio as a new quality metric of colonoscopy, endoscopists with cecal intubation time/withdrawal time $<1$ detected significantly more adenomas compared to endoscopists with ratios $>1$. Nevertheless, the relationship between the absolute insertion time and ADR was not directly addressed in the study [7].

Of interest is the relationship between insertion time and withdrawal time in the three aforementioned studies. In the two observational studies where the endoscopists were unaware that they were being observed and theoretically 
not influenced by the effects of observation during colonoscopy, an inverse association between insertion time and withdrawal time was observed [5, 7]. Conversely, in the RCT study, insertion time was not associated with withdrawal time [6]. In the absence of being observed in the "real-life" situation, it is reasonable to expect that the colonoscopists, tired and time-pressed after a prolonged insertion, are likely to remove the colonoscope faster and therefore compromise the quality of inspection. Yet, the fact that a longer insertion time was still linked to a lower ADR despite a lack of association of insertion time and withdrawal time in the RCT study suggested that other factors might contribute.

Given that most published studies found that a longer insertion time had a detrimental effect on ADR, the report by Fritz et al. in this issue of Digestive Diseases and Sciences appears to be an exceptional finding [8]. The authors conducted a retrospective analysis of 1303 patients enrolled in four RCTs to evaluate the association between cecal insertion time and adenoma detection. In contrast to the aforementioned studies [5, 6], they found that a prolonged cecal insertion time was not associated with a decreased ADR or a decreased mean number of detected adenomas or advanced adenomas. Withdrawal time increased when the mean cecal insertion time increased $(p<0.001)$, which is in stark contrast to the inverse association observed in previous studies, and may partly explain why longer insertion time was not associated with a lower ADR in the current study. It appears that the issue of reduced ADR by a longer insertion time could be overcome by a relatively longer withdrawal timethat is to say, all you need is patience.

Moreover, the current study found that when cecal insertion times were longer than withdrawal times, i.e., insertion time/withdrawal time $>1$, the number of detected adenomas and advanced adenomas decreased. By incorporating the insertion time into the equation, the ratio takes the association between withdrawal time and ADR one step further. It may be possible to improve ADR by decreasing the ratio through either decreasing the insertion time or increasing the withdrawal time; additional studies are warranted to confirm this finding. Theoretically, the maneuvers that help shorten the insertion time, such as loop reduction and scope straightening, might also make the withdrawal inspection easier by keeping the colon straight. The extra time gained from a speedy insertion can be redistributed to withdrawal phase, allowing for a more meticulous examination. On the other hand, when the intubation is difficult and prolonged, instead of removing the scope quickly to compensate for the lost time, a slow withdrawal and meticulous examination will be imperative in order to avoid missing polyps.

One of the important findings of the current study is that more advanced adenomas were found when the insertion/withdrawal time was $<1$, similar to the finding by Von Renteln et al. in that a longer cecal insertion time was associated with a decreased detection of advanced adenomas [6]. Advanced adenomas have a high chance of evolving into cancers with a cumulative risk of $24 \%$ at 20 years [9]; their presence is also linked to a higher risk of subsequent ICs according to a recently published prospective cohort study [10].

The current study has a number of strengths. It has a relatively large sample size, and its data were collected prospectively. A total of 28 colonoscopists were enlisted, which improved the generalizability of the results. The mean withdrawal time for patients without polypectomy was $10.9 \pm 6.2 \mathrm{~min}$; the overall ADR was $45 \%$ and advanced ADR was $12 \%$, all of which meet the current recommended standards of care. Nevertheless, the study also has several limitations. It is a retrospective analysis of data collected in four prospective RCTs at a single tertiary care center. Although the mean withdrawal time without polypectomy was provided, the analysis was performed using total withdrawal time (time for polypectomy included). As noted by the authors, because adenoma detection was the focus of study designs of the original four RCTs, the "Hawthorne effect" (behavior alteration due to observation) might be present and the results might differ from real-life situations if the endoscopists are not under the influence of observation.

In conclusion, the authors of the current study are to be commended for their convincing support of the hypothesis that cecal insertion time/withdrawal time $<1$ was associated with an increased number of detected adenomas and advanced adenomas, whereas a shorter insertion time per se was not. Nevertheless, the results are still far from conclusive. Whether the ratio is a useful predictor of ADR and thus a colonoscopic quality metric warrants further investigation. Notably, the unique finding of a positive association between insertion time and withdrawal time not only suggested that the detrimental effect of prolonged insertion time on ADR could be overcome by a longer withdrawal time, but also 
Table 1 Summary of recently published studies addressing the relationship between cecal insertion time and adenoma detection

\begin{tabular}{|c|c|c|c|}
\hline Study & Sample size and study design & $\begin{array}{l}\text { Relationship between cecal } \\
\text { insertion time and withdrawal } \\
\text { time }\end{array}$ & Impact on adenoma detection \\
\hline Benson et al. [7] & $\begin{array}{l}550 \text { consecutive average-risk subjects } \\
\text { undergoing screening colonoscopies }\end{array}$ & Inverse association & $\begin{array}{l}\text { Endoscopists with insertion time-to-with- } \\
\text { drawal time ratios of }<1 \text { detected more } \\
\text { adenomas compared to endoscopists with } \\
\text { ratios }>1\end{array}$ \\
\hline Yang et al. [5] & $\begin{array}{l}\text { Cross-sectional study of } 12,679 \text { consecu- } \\
\text { tive subjects }\end{array}$ & Inverse association & $\begin{array}{l}\text { A shorter insertion time was associated } \\
\text { with an increased detection rate of small } \\
\text { colorectal adenomas }<5 \mathrm{~mm}\end{array}$ \\
\hline von Renteln et al. [6] & $\begin{array}{l}1043 \text { patients in an randomized controlled } \\
\text { trial on cap-assisted colonoscopy }\end{array}$ & No association & $\begin{array}{l}\text { A longer insertion time was associated with } \\
\text { a decreased detection of adenomas and } \\
\text { advanced adenomas }\end{array}$ \\
\hline Fritz et al. [8] & $\begin{array}{l}\text { Retrospective analysis of } 1303 \text { subjects } \\
\text { enrolled in four randomized controlled } \\
\text { trials undergoing screening and surveil- } \\
\text { lance colonoscopy }\end{array}$ & Direct association & $\begin{array}{l}\text { Insertion time/withdrawal time }>1 \text { was } \\
\text { associated with decreased detected adeno- } \\
\text { mas and advanced adenomas/patient, but a } \\
\text { longer insertion time per se was not }\end{array}$ \\
\hline
\end{tabular}

raised the issue of the Hawthorne effect and the applicability of the results to real-life clinical practice (Table 1).

\section{References}

1. Corley DA, Jensen CD, Marks AR, et al. Adenoma detection rate and risk of colorectal cancer and death. $N$ Engl J Med. 2014;370:1298-1306.

2. Barclay RL, Vicari JJ, Doughty AS, Johanson JF, Greenlaw RL. Colonoscopic withdrawal times and adenoma detection during screening colonoscopy. $N$ Engl J Med. 2006;355:2533-2541.

3. Lee TJ, Blanks RG, Rees CJ, et al. Longer mean colonoscopy withdrawal time is associated with increased adenoma detection: evidence from the Bowel Cancer Screening Programme in England. Endoscopy. 2013;45:20-26.

4. Hsieh YH, Kuo CS, Tseng KC, Lin HJ. Factors that predict cecal insertion time during sedated colonoscopy: the role of waist circumference. J Gastroenterol Hepatol. 2008;23:215-217.
5. Yang MH, Cho J, Rampal S, et al. The association between cecal insertion time and colorectal neoplasm detection. BMC Gastroenterol. 2013;13:124.

6. von Renteln D, Robertson DJ, Bensen S, Pohl H. Prolonged cecal insertion time is associated with decreased adenoma detection. Gastrointest Endosc. 2017;85:574-580.

7. Benson ME, Reichelderfer M, Said A, Gaumnitz EA, Pfau PR. Variation in colonoscopic technique and adenoma detection rates at an academic gastroenterology unit. Dig Dis Sci. 2010;55:166-171.

8. Fritz CDL, Smith ZL, Elsner J, Hollander T, Early D, Kushnir V. Prolonged cecal insertion time is not associated with decreased adenoma detection when a longer withdrawal time is achieved. Dig Dis Sci. (Epub ahead of print). https://doi.org/10.1007/s1062 0-018-5100-x.

9. Stryker SJ, Wolff BG, Culp CE, Libbe SD, Ilstrup DM, MacCarty RL. Natural history of untreated colonic polyps. Gastroenterology. 1987;93:1009-1013.

10. Click B, Pinsky PF, Hickey T, et al. Association of colonoscopy adenoma findings with long-term colorectal cancer incidence. JAMA. 2018;319:2021-2031. 